\title{
Study of Artificial Boundary Problems based on Numerical Manifold Method
}

\author{
LI Yang 1,2,a , ZHENG Hong ${ }^{1,2, b}$ \\ 1State Key Laboratory of Geomechanics and Geotechnical Engineering, Institute of Rock and \\ Soil Mechanics, Chinese Academy of Sciences, Wuhan, P.R. China \\ 2University of Chinese Academy of Sciences, Beijing, P.R. China \\ asmartkokoro@qq.com, bhzheng@whrsm.ac.cn
}

\begin{abstract}
Keywords: NMM; artificial boundary methods; viscous boundary; transmitting boundary; stress wave

Abstract. The article uses and compares effects of two artificial boundary methods when they are applied to problems of stress waves in NMM methods. Starting from D'Alembert's solution, the article finds another way to exerting viscous boundary. By modeling in NMM method and exerting fixed boundary, viscous boundary and transmitting boundary in the research of stress wave propagating in a thin rod, after observing the stress, displacement and velocity, it is discovered these artificial boundaries both have the ability to absorb the stress wave. Especially, the 2nd transmitting boundary performs a great absorbing efficiency of $99.6 \%$.
\end{abstract}

\section{Introduction}

Numerical Manifold Method(NMM) is a numerical method based on finite covers [1]. It reaches a unified solution for the problems from rock and soil mechanics. Since it was proposed by Dr.SHI in 1990s, many scholars did a lot of researches and applications and gained great success in static problems. However, researches of dynamics using NMM are still not enough.

In the process of solving typical dynamic problems of infinite domain, the artificial boundary method is usually used. In 1968, Alterman used the remote artificial boundary of exerting artificial boundary far enough from the main calculating areas [2], it greatly reduced the difficulty of calculating problems of dynamic infinite domain. But this method caused a great scale of calculation which is unaffordable. Then Lysmer and Kuhlemeyer proposed the viscous boundary which is still widely used nowadays in 1969 [3]. At the same time, Smith W. introduced the superimposed boundary by taking the average of solutions of the Dirichlet and Newmann boundaries [4]. In China, Liao introduced the transmitting boundary in 1996 [5]. He deduced a new and concise artificial boundary by dispersing the D'Alembert's solution.

To seek for the artificial boundary fitting to the NMM, this paper imports and compares several artificial boundaries.

\section{Artificial Boundaries in NMM}

2.1 Brief Introduction to NMM. NMM is composed of mainly four parts: the cover systems, the partition of unity, the NMM space and the variational formulation [1]. The most featured part are the cover systems and the partition of unity.

The NMM cover systems have two parts: the math cover(MC) and the physical cover(PC). MC is the basic cover and due to the boundaries and discontinuities, the MC is cut into several pieces of PCs. The relationships between the two cover can be expressed below:

$$
\bigcup_{j=1}^{n_{i}} P_{i-j} \subseteq M_{i}
$$

$n_{i}$ is the number of PC from the math cover $M_{i}$. 
In NMM, the problem domain is covered by m math covers and math cover $M_{i}$ can be cut into $n_{i}$ physical covers. Each physical cover has a corresponding weight function which must satisfy the partition of unity. That is in a point $\mathrm{z}$, the sum of the weight function should be:

$$
\sum_{i=1}^{m} \sum_{j=1}^{n_{i}} \omega_{i-j}=1
$$

Here, $\omega_{i-j}$ represents the weight function of $P_{i-j}$ and it also satisfies:

$$
\begin{cases}\omega_{i-j}=0 & \text { if } z \notin P_{i-j} \\ \omega_{i-j} \geq 0 & \text { if } z \in P_{i-j}\end{cases}
$$

Then the displacement of point $\mathrm{z}$ can be expressed:

$$
u(z)=\sum \omega_{i-j} u_{i-j}
$$

$u_{i-j}$ is the displacement of $P_{i-j}$. It is usually obtained by the Taylor expansion:

$$
u_{i-j}(z)=u_{i-j}^{0}+\sum_{i=1}^{n} \frac{1}{i !}(\Delta z \bullet \nabla)^{i} u_{i-j}, \quad z \in P_{i-j}
$$

In the practical application, we always take $u_{i-j}(z)=u_{i-j}^{0}$. Then we get:

$$
u(z)=\sum \omega_{i-j} u_{i-j}^{0}
$$

Here we see the variables that need to be calculated are the displacements of the PCs. And the shape function is composed of the weight function. These things lead it to the advantages of NMM. That is:

1) The NMM can handle problems with strong discontinuity because the MC can be cut as whatever it need to be.

2) The NMM has little mesh dependence as the basics, PCs can be superimposed and it also means the displacement function can be set without consideration of the harmony at the boundary of the PCs.

2.2 The Fixed Boundary. Exerting the fixed boundary in NMM is the easiest way of solving the infinite domain problems. However, it performs badly. Here, this boundary is used for comparison.

The penalty function method is used to exert the fixed boundary. By choosing a rational $k$ as the penalty, the displacement of boundary is almost fixed to 0 . Then we can get the potential energy of the boundary:

$$
\Pi=\frac{k}{2} d_{i}^{T} N^{T} N d_{i}
$$

Here, matrix $N, d_{i}$ are shape function and displacements of NMM respectively. Use the variational method and the stiffness matrix is got:

$$
k N^{T} N \rightarrow[K]
$$

2.3 The Viscous Boundary. The viscous boundary absorbs the energy of the stress wave by exerting damper in normal and tangential directions. Since this method was introduced, it has been widely used in finite element method(FEM) and proven as a very effective artificial boundary method. Yang. Etc. explored the effect of using the viscous boundary in NMM firstly and G.F. Zhao used this boundary to calculate the propagation of stress wave in infinite rocks with fracture $[7,8]$. They both found that the viscous boundary was useful in NMM, but the efficiency was not always perfect.

The viscous boundary method exerts normal and tangential viscous spring and it provides viscous force of velocity correlation as fellow:

$$
\left\{\begin{array}{l}
\sigma_{n}=-n_{1} \rho c_{p} v_{n} \\
\tau_{n}=-n_{2} \rho c_{s} v_{s}
\end{array}\right.
$$

Here, $n_{1}, n_{2}$ are the absorbing coefficients of the viscous boundary, which is used to adjust the viscous force. These two coefficients are usually valued as $n_{1}=n_{2}=1$ in the finite element method. But in NMM, what the values of the two coefficients should be is still not determined. By numerical 
experiment, we found that the two coefficients should be adjusted as the external conditions change such as the material properties and the time steps. $v_{n}, v_{s}$ are the normal and tangential velocities at the artificial boundary.

$c_{p}, c_{s}$ are the velocity of the $\mathrm{P}$ wave and the $\mathrm{S}$ wave in the chosen material, value as:

$$
\left\{\begin{array}{c}
c_{p}=\sqrt{(K+4 G / 3) / \rho} \\
c_{s}=\sqrt{G / \rho}
\end{array}\right.
$$

$\mathrm{K}$ is the bulk modulus of the material. $\mathrm{G}$ is the shear modulus of the material. $\rho$ is the density of the chosen material.

Then, the viscous forces acting on the artificial boundary in normal and tangential directions can be expressed as:

$$
\left\{\begin{array}{l}
f_{n} \\
f_{s}
\end{array}\right\}=-\int_{0}^{l} \rho\left[\begin{array}{cc}
n_{1} c_{p} & 0 \\
0 & n_{2} c_{s}
\end{array}\right]\left\{\begin{array}{l}
v_{n} \\
v_{s}
\end{array}\right\} d l
$$

$\mathrm{L}$ is the integral length of the single damper in the viscous boundary.

After the coordinate transformation, we can get the damp force on the viscous boundary and then lead to the potential energy of every single damper:

$$
\Pi=-\int_{0}^{l} \rho u^{T}\left[\begin{array}{cc}
n_{1} c_{p} & 0 \\
0 & n_{2} c_{s}
\end{array}\right] \times\left[\begin{array}{cc}
\sin \alpha & -\cos \alpha \\
\cos \alpha & \sin \alpha
\end{array}\right]\left\{\begin{array}{l}
v_{x} \\
v_{y}
\end{array}\right\} d l
$$

But here we can notice a problem. The essential variable, the velocity $v_{n+1}$ on the artificial boundary is not known when we get to calculate the potential energy at each time step. So a rational way to calculate $v_{n+1}$ determines the accuracy of the viscous boundary and using $v_{n}$ as $v_{n+1}$ yields a result of terrible precision. From the Newmark Method we can see that $v_{n+1}$ can be obtained from velocity and acceleration of the last time step:

$$
\begin{gathered}
v_{n+1}=v_{n}+\left[(1-\delta) a_{n}+\delta a_{n+1}\right] \Delta t \\
a_{n+1}=\frac{1}{\alpha \Delta t^{2}}\left(s_{n+1}-s_{n}\right)-\frac{1}{\alpha \Delta t} v_{n}-\left(\frac{1}{2 \alpha}-1\right) a_{n}
\end{gathered}
$$

Here, $s_{n+1}$ is the accumulative displacement in the $n+1$ time step. The difference $s_{n+1}-s_{n}$ is the displacement $u_{n+1}$ in the $\mathrm{n}+1$ time step. $\alpha, \delta$ are the Newmark parameters that are usually valued as 0.25 and 0.5 . $\Delta t$ was the length of one single time step.

Plug formula (13) into (14), we get the expression of $v_{n+1}$ :

$$
v_{n+1}=\frac{2}{\Delta t} u_{n+1}-v_{n}
$$

Then put (15) into (12) and do some simplification, we can get the stiffness matrix and add it to the total stiffness matrix:

$$
\left\{\begin{array}{c}
\int_{0}^{l} \frac{2 \rho}{\Delta t}[T]^{T}\left[\begin{array}{cc}
n_{1} c_{p} & 0 \\
0 & n_{2} c_{s}
\end{array}\right]\left[\begin{array}{cc}
\sin \alpha & -\cos \alpha \\
\cos \alpha & \sin \alpha
\end{array}\right][T] d l \rightarrow K \\
\int_{0}^{l} \rho[T]^{T}\left[\begin{array}{cc}
n_{1} c_{p} & 0 \\
0 & n_{2} c_{s}
\end{array}\right]\left[\begin{array}{cc}
\sin \alpha & -\cos \alpha \\
\cos \alpha & \sin \alpha
\end{array}\right] v d l \rightarrow F
\end{array}\right.
$$

2.4 The Transmitting Boundary. The transmitting boundary is a very concise artificial boundary and it is very easy to be applied to the numerical methods including NMM. But we need to do some investigation of the performance of this boundary in NMM to see if it is good enough. The $n^{\text {th }}$ transmitting boundary is:

$$
\begin{gathered}
u_{0}^{p+1}=\sum_{j=1}^{n}(-1)^{j+1} C_{j}^{n} u_{j}^{p+1-j} \\
C_{j}^{m}=\frac{m !}{(m-j) ! j !}
\end{gathered}
$$


Because of the complexity when using high order transmitting boundary, we choose the first and second order transmitting boundary:

$$
\begin{gathered}
u_{0}^{p+1}=u_{1}^{p} \\
u_{0}^{p+1}=2 u_{1}^{p}-u_{2}^{p-1}
\end{gathered}
$$

Also, we use the penalty function method to exert the transmitting boundary. Then we can get the potential energy of the transmitting boundary:

$$
\Pi=\frac{k}{2}\left(u-u_{0}^{p+1}\right)^{2}
$$

After the variation, the stiffness matrix is got and added to the total stiffness matrix:

$$
\left\{\begin{array}{l}
k N^{T} N \rightarrow[K] \\
k N u_{0}^{p+1} \rightarrow[F]
\end{array}\right.
$$

Here, the $u_{0}^{p+1}$ is obtained from the transmitting boundary.

From the formula above, we can see the application of the transmitting boundary into NMM is very convenient.

\section{Numerical Example}

We choose a thin rock rod which is $0.03 \mathrm{~m}$ wide and has infinite length. The artificial boundaries are exerted at the length of $1 \mathrm{~m}$. The parameters are chosen as below: the density is $2600 \mathrm{~kg} / \mathrm{m}^{3}$; the Poisson ratio is 0.25 ; the elastic modulus is $50 \mathrm{GPa}$; the length of the time step is $5 \times 10^{-6} \mathrm{~s}$.

From the material properties, the $\mathrm{P}$ wave velocity is $5430.21 \mathrm{~m} / \mathrm{s}$ and the $\mathrm{S}$ wave velocity is $2773.50 \mathrm{~m} / \mathrm{s}$.

The rod is covered by math covers of regular hexagon with side length $0.015 \mathrm{~m}$. The coordinate of the observation point is $(0.65 \mathrm{~m}, 0.015 \mathrm{~m})$. The coordinate of the input point is $(0,0.015 \mathrm{~m})$ and the input stress wave is:

$$
\left\{\begin{array}{cc}
p=0.9(n-1) & n \leq 5 \\
p=8.1-0.9 n & 5<t \leq 9
\end{array}\right.
$$

Here, $\mathrm{n}$ is the number of the time step. The unit of $\mathrm{p}$ is MPa. Then we can get the stress and displacement of all the artificial boundaries at the observation point as fellow:

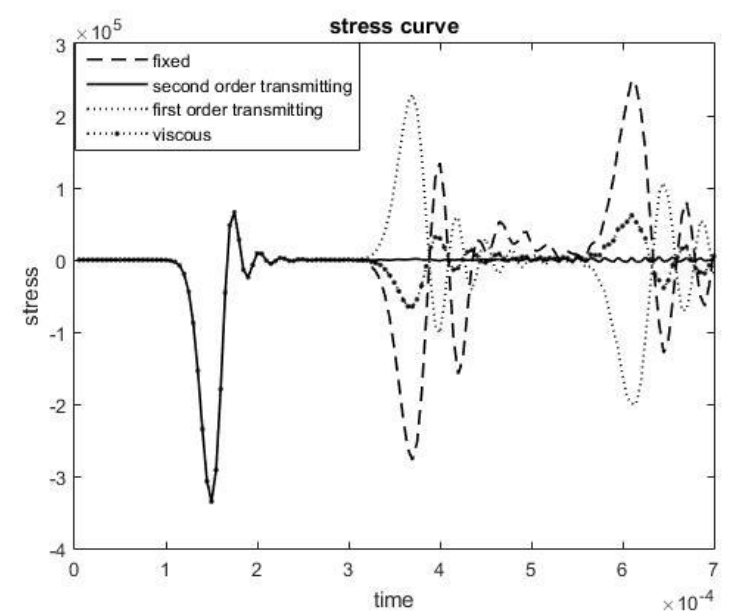

Fig.1 Stress curve of 4 artificial boundaries

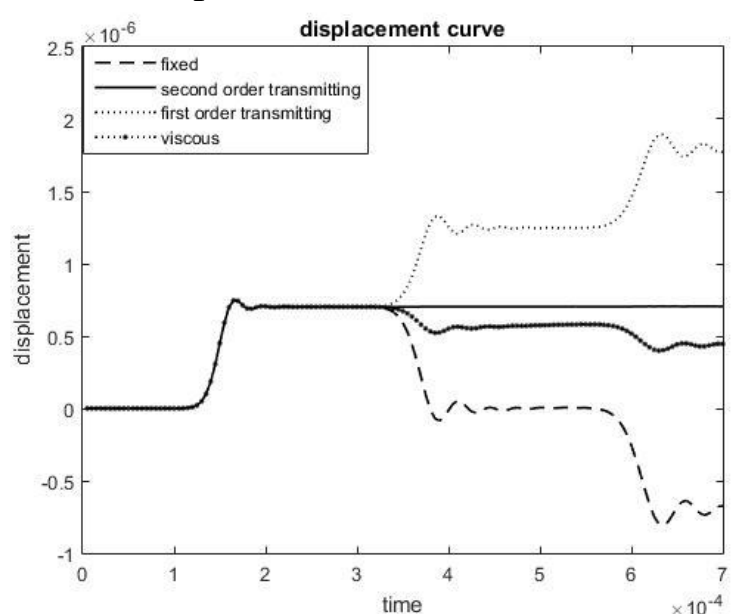

Fig.2 Displacement curve of 4 artificial boundaries

From the two figures we can see that the fixed boundary leads to obvious reflection which is almost the same as the input stress wave. The viscous boundary performs much better. At the time of $3.75 \times 10^{-4} \mathrm{~s}$, the reflection reaches the climax of $5.48 \times 10^{4} \mathrm{~Pa}$. It shows this boundary has an absorbing efficiency of $84.8 \%$. The displacement goes lower after the reflection wave passing by. It illustrates the viscous boundary has the capability of reducing the energy of the stress wave in its 
propagating direction and damping the displacement. But the efficiency in NMM isn't very high and it's changing with the damping parameter.

Though the first order transmitting boundary shows poor performance in this case, the second order transmitting boundary gets an absorbing efficiency of $99.6 \%$ : at the climax point, the stress is only $1.44 \times 10^{3} \mathrm{~Pa}, 0.4 \%$ of the original value. After the stress wave passing by, the displacement also becomes stable. That means, in NMM, the second transmitting boundary performs perfectly with almost zero reflection.

\section{Summary}

1)In this paper, mainly three artificial boundaries were compared. It results that the viscous boundary has the ability to absorb the stress wave in NMM, but it can't perform as well as it does in finite element method.

2)The second order transmitting boundary shows a great absorption efficiency as high as $99.6 \%$. The transmitting boundary of the lower order can't make it to a good result and the higher order is too complex. That means the second transmitting boundary is very fit for the NMM.

\section{Acknowledgements}

This work was financially supported by the National Natural Science Foundation of China, under the Grant No.51538001.

\section{References}

[1] Zheng H, Liu F, Li CG. The MLS-based numerical manifold method with applications to crack analysis[J]. Int J Fract (2014) 190:147-166.

[2] SHI Gen-hua, PEI Jue-min. Numerical Manifold Method(NMM) and Discontinuous Deformation Analysis(DDA)[D]. Beijing: Tsinghua University Press, 1997. (in Chinese)

[3] Z S Alterman, E Isaacson. A method for calculating frontal motion I. dimensional tests[J]. Journal of computational physics, 1969,4(1): 67-96.

[4] Lysmer J, Kuhlemeyer RL. Finite element method accuracy for wave propagation problems[J]. Journals of the Soil Mechanics and Foundations Division ASCE 1973; 99(5):421-427.

[5] Smith W. A non-reflecting plane boundary for wave propagation problems[J]. Journal of Computational Physics, 1973,15: 492-503.

[6] Liao Zhen-peng. Introduction to Wave Motion Theories in Engineering (the second edition) [D]. Beijing: Science Press, 2002. (in Chinese)

[7] QIAN Ying, YANG Jun. Primary Study on Viscous Boundary in Numerical Manifold Method[J]. Chinese Journal of Computational Mechanics, 2009,26(5): 757-760. (in Chinese)

[8] G.F. Zhao, X.B. Zhao and J.B. Zhu. Application of the numerical manifold method for stress wave propagation across rock masses[J]. International Journal for Numerical and Analytical Methods in Geomechanics.

[9] FU Xiao-dong, SHENG Qian, ZHANG Yong-hui. Investigation on Artificial Boundary Problem in Discontinuous Deformation Analysis Method[J]. Chinese Journal of Rock Mechanics and Engineering, 2015,34(5): 986-993. (in Chinese)

[10] Karl F. Graff. Wave Motion in Elastic Solids[D]. New York: Dover Publications, INC, 1991.

[11] NING You-jun, YANG Jun, CHEN Peng-wan. Two Non-Reflecting Boundary Conditions in DDA Method[J]. Engineering Mechanics, 2010,27(4): 19-23. (in Chinese) 